\title{
Predictive Transient-Following Control of Shunt and Series Active Power Filters
}

\author{
John H. Marks, Student Member, IEEE, and Tim C. Green, Member, IEEE
}

\begin{abstract}
A novel technique is presented for generation of a contemporary estimate of the fundamental component of the distorted input current or voltage to an uncontrolled three-phase bridge rectifier with a DC link smoothing filter. This allows for accurate calculation of cancellation references for series and shunt active power filters (APF) operating under steady-state and transient conditions. Improved transient performance allows for reduction of the power rating and control system bandwidth of an APF.

An artificial neural network (ANN) predictor has been used to directly calculate the mean $d q$-axis input to the rectifier without filtering. This is a critical stage in separating harmonic distortion from fundamental current or voltage. The technique is developed using simulation data for both series and shunt APFs and validated with experimental results. The predictive harmonic identifier shows good steady-state performance and excellent transient performance that far exceeds that of a conventional identifier using time-domain or frequency-domain filtering.
\end{abstract}

Index Terms-Active filters, neural networks, predictive control, transient response.

\section{INTRODUCTION}

A large number of nonlinear loads are used in applications at various power levels. Examples of nonlinear loads include variable speed drives, lighting systems, switch mode power supplies and many more types besides. These devices can cause distortion to the mains supply by drawing harmonic currents and voltages. Traditionally tuned passive filters have been used to attenuate the harmonic distortion. The use of passive filters is not desirable as they are bulky, de-tune with age and can resonate with the supply impedance. In order to overcome the limitations of passive filtering techniques, there has been much research activity in the use of active power filters (APF) to mitigate the harmonic distortion produced by semiconductor switched loads. The APF has may advantages over using passive filters. The APF can be smaller, cheaper, more versatile, better damped, more selective and less prone to failure through component drift than its passive counterpart. It can also be used to compensate reactive power.

When using an APF to cancel a harmonic distorting load the type of distortion must be classified as either current-type or voltage-type [1]. The example harmonic load considered in this paper is a three-phase uncontrolled diode bridge rectifier. The

Manuscript received November 23, 2000; revised May 1, 2001. Recommended by Associate Editor J. H. R. Enslin.

The authors are with the Imperial College of Science, Technology and Medicine, Department of Electrical and Electronic Engineering, London SW7 2BT, U.K. (e-mail: j.h.marks@ic.ac.uk; t.green@ic.ac.uk).

Publisher Item Identifier 10.1109/TPEL.2002.800970.

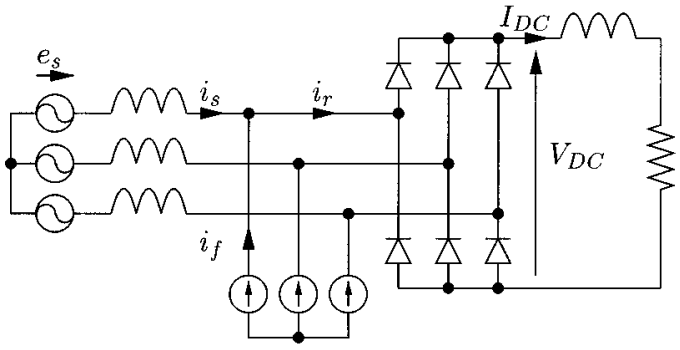

Fig. 1. Three-phase rectifier with inductive dc link filter and shunt APF.

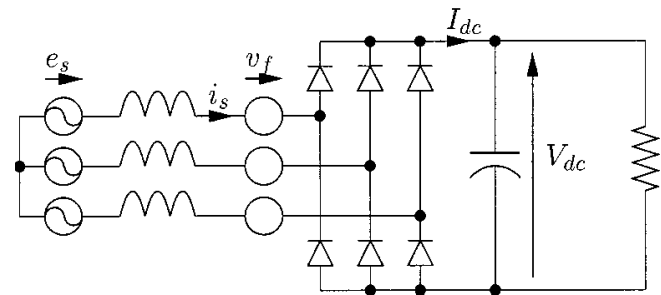

Fig. 2. Three-phase rectifier with capacitive dc link filter and series APF.

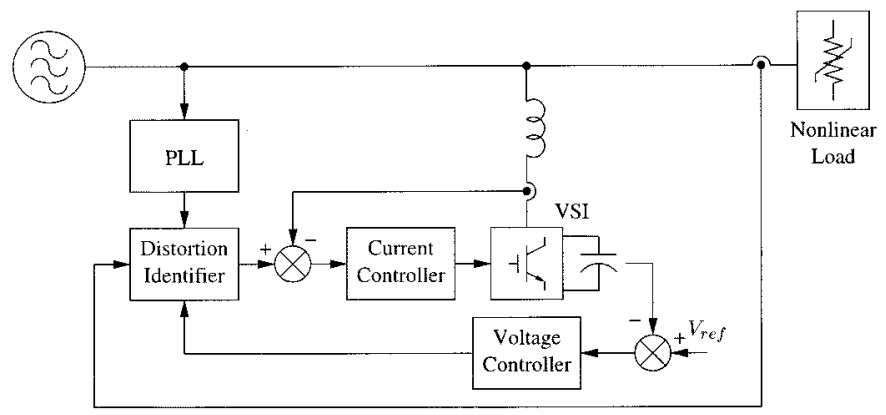

Fig. 3. Complete shunt APF control system.

DC link smoothing filter determines the type of harmonic distortion produced. In turn, the type of distortion determines the type of APF required [1]. Fig. 1 shows a shunt APF used to cancel harmonic current distortion. Fig. 2 shows a series APF used to cancel harmonic voltage distortion. These two situations are duals of each other. Both are considered in this paper. Throughout the paper the shunt-APF/current-distortion case is the main description with the series case following in parentheses.

\section{CONVENTiOnAl APF CONTROL}

Fig. 3 shows an example of a shunt APF. The correction current that will cancel the harmonic content of the load current is supplied by a voltage source inverter (VSI). There are three main tasks for the controller. 


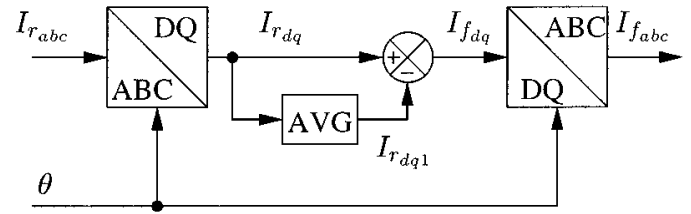

Fig. 4. Conventional low-pass APF harmonic identifier.

1) To identify the harmonic content and form a synchronised reference.

2) Provide closed-loop control to force the current of the VSI to follow the reference.

3) To regulate the VSI dc link voltage in the face of power losses in the VSI and erroneous real power (fundamental frequency) exchange with the line.

This paper reassesses the first of these control tasks and assumes the other two are tackled in the normal way.

Conventionally, time-domain [2]-[5] or frequency-domain [6] filtering techniques have been used to identify the harmonic component of the distorted signal. This can either be done directly, or indirectly by obtaining the fundamental frequency component and subtracting it from the instantaneous value. The identification can be performed in a number of different reference frames (ABC, $\alpha \beta 0$, dq0, pq0) [3], [7]. This method has been proven to work effectively under steady-state operating conditions. However, under transient conditions when the fundamental component of the distorted signal is varying dynamically, the conventional filtering techniques exhibit significant delays in tracking the fundamental.

For the direct method this results in incomplete cancellation of harmonic distortion. In the case of the indirect method, an error in the estimated fundamental frequency component will result in a residual fundamental frequency component in the cancellation reference. This will cause the APF to source/sink real power. Therefore, to prevent damage to the APF, the power converter and energy storage must be overrated. It is difficult to implement a fast response energy balance control loop because it interacts with the cancellation process and causes distortion.

Fig. 4 shows a block diagram of a conventional harmonic identifier. The identifier operates in the synchronous rotating reference frame (dq0) using the indirect method. This identifier is used as a benchmark by which to gauge the performance of the predictive identifier.

The choice of filter used determines the performance of the system. The filter used is a second order Butterworth low-pass infinite impulse response (IIR) design with a $10 \mathrm{~Hz}$ cut-off frequency. This type of filter is chosen as it gives good transient response, while maintaining acceptable attenuation of the harmonics present.

\section{RELATIONSHIP BETWEEN AC AND DC QuANTITIES OF A RECTIFIER}

The predictive harmonic identifier is based on the assumption that there is a relationship between the mean value of the smoothed dc link current (voltage) of a three-phase rectifier with inductive (capacitive) dc link smoothing filter and its mean $d q$-axis input counterparts. In order to validate this assumption a relationship is analytically derived for an ideal three-phase six-pulse rectifiers with either inductive or capacitive dc link smoothing filters. The derivation assumes the following operating conditions.

- The smoothed de link output current (voltage) is constant.

- Diode commutation is instantaneous.

The derivation is carried out in three stages. Firstly, a relationship between the mean $d q$-axis rectifier input currents (voltages) and the peak supply current (voltage) is found. Secondly, the relationship between the smoothed dc link current (voltage) and the peak ac input current (voltage) is derived by Fourier analysis. Finally, the two expressions are substituted to yield the relationship between the mean $d q$-axis input currents (voltages) and the dc link current (voltage).

\section{A. Current Rectifier}

Fig. 5 shows normalized ideal current waveforms for a single phase of a three-phase rectifier with inductive dc link filter and shunt APF (Fig. 1).

The relationship between the peak fundamental ac input current and the mean $d q$-axis values is found using the familiar power invariant $d q$ transformation [8]. The transformation matrix used is the combined power invariant Clarke and Park transformations

$$
\begin{aligned}
{\left[\begin{array}{l}
\bar{I}_{d} \\
\bar{I}_{q}
\end{array}\right]=} & \sqrt{\frac{2}{3}}\left[\begin{array}{lll}
\cos \omega t & \cos \left(\omega t-\frac{2 \pi}{3}\right) & \cos \left(\omega t+\frac{2 \pi}{3}\right) \\
\sin \omega t & \sin \left(\omega t-\frac{2 \pi}{3}\right) & \sin \left(\omega t+\frac{2 \pi}{3}\right)
\end{array}\right] \\
& \cdot\left[\begin{array}{c}
\hat{I}_{s} \cos (\omega t+\phi) \\
\hat{I}_{s} \cos \left(\omega t-\frac{2 \pi}{3}+\phi\right) \\
\hat{I}_{s} \cos \left(\omega t+\frac{2 \pi}{3}+\phi\right)
\end{array}\right] \\
= & \sqrt{\frac{3}{2}}\left[\begin{array}{c}
\cos \phi \\
\sin \phi
\end{array}\right] \hat{I}_{s} .
\end{aligned}
$$

The relationship between the dc link current and the peak fundamental component of the ac input current is derived by Fourier analysis of the load current waveform

$$
\begin{aligned}
\hat{I}_{s} & =\frac{1}{\pi} \int_{0}^{2 \pi} I_{r}(\theta) \sin \theta d \theta \\
& =\frac{I_{d c}}{\pi}\left\{\int_{\pi / 6}^{5 \pi / 6} \sin \theta d \theta-\int_{7 \pi / 6}^{11 \pi / 6} \sin \theta d \theta\right\} \\
& =\frac{I_{d c}}{\pi}\left\{-[\cos \theta]_{\pi / 6}^{5 \pi / 6}+[\cos \theta]_{7 \pi / 6}^{11 \pi / 6}\right\} \\
& =\frac{2 \sqrt{3}}{\pi} I_{d c} .
\end{aligned}
$$

The relationship between the dc link current and the mean $d q$-axis input currents is derived by substituting (2) into (1)

$$
\begin{aligned}
{\left[\begin{array}{l}
\bar{I}_{d} \\
\bar{I}_{q}
\end{array}\right] } & =\sqrt{\frac{3}{2}}\left[\begin{array}{c}
\cos \phi \\
\sin \phi
\end{array}\right] \frac{2 \sqrt{3}}{\pi} I_{d c} \\
& =\frac{3 \sqrt{2}}{\pi}\left[\begin{array}{c}
\cos \phi \\
\sin \phi
\end{array}\right] I_{d c} .
\end{aligned}
$$

\section{B. Voltage Rectifier}

Fig. 6 shows normalized ideal phase voltage waveforms for a single phase of a three-phase rectifier with capacitive dc link filter and series APF (Fig. 2). 

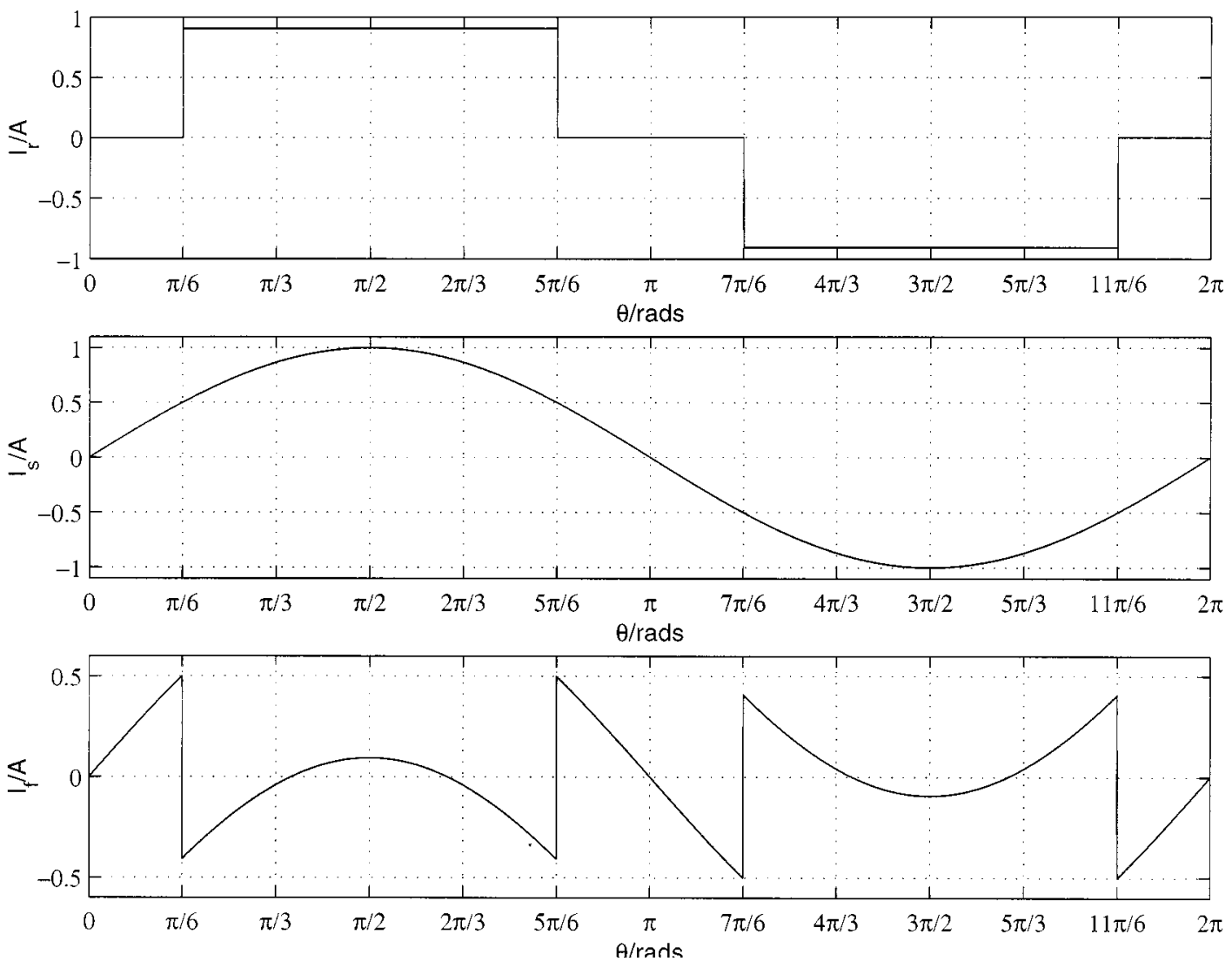

Fig. 5. Normalized ideal current waveforms for inductive rectifier with shunt APF.

The relationship between the peak fundamental ac input phase voltage and the mean $d q$-axis values is found using the power invariant $d q$ transformation

$$
\begin{aligned}
{\left[\begin{array}{l}
\bar{V}_{d} \\
\bar{V}_{q}
\end{array}\right]=} & \sqrt{\frac{2}{3}}\left[\begin{array}{lll}
\cos \omega t & \cos \left(\omega t-\frac{2 \pi}{3}\right) & \cos \left(\omega t+\frac{2 \pi}{3}\right) \\
\sin \omega t & \sin \left(\omega t-\frac{2 \pi}{3}\right) & \sin \left(\omega t+\frac{2 \pi}{3}\right)
\end{array}\right] \\
& \cdot\left[\begin{array}{c}
\hat{V}_{s} \cos (\omega t+\phi) \\
\hat{V}_{s} \cos \left(\omega t-\frac{2 \pi}{3}+\phi\right) \\
\hat{V}_{s} \cos \left(\omega t+\frac{2 \pi}{3}+\phi\right)
\end{array}\right] \\
& =\sqrt{\frac{3}{2}}\left[\begin{array}{l}
\cos \phi \\
\sin \phi
\end{array}\right] \hat{V}_{s} .
\end{aligned}
$$

The relationship between the dc link voltage and the peak fundamental component of the ac input phase voltage is derived by Fourier analysis of the load phase voltage waveform

$$
\begin{aligned}
& \hat{V}_{s}= \frac{1}{\pi} \int_{0}^{2 \pi} V_{r}(\theta) \sin \theta d \theta \\
&=\frac{V_{d c}}{3 \pi}\left\{\int_{0}^{\pi / 3} \sin \theta d \theta+2 \int_{\pi / 3}^{2 \pi / 3} \sin \theta d \theta\right. \\
& \quad+\int_{2 \pi / 3}^{\pi} \sin \theta d \theta-\int_{\pi}^{4 \pi / 3} \sin \theta d \theta \\
&\left.\quad-2 \int_{4 \pi / 3}^{5 \pi / 3} \sin \theta d \theta-\int_{5 \pi / 3}^{2 \pi} \sin \theta d \theta\right\} \\
&=\frac{V_{d c}}{3 \pi}\left\{-[\cos \theta]_{0}^{\pi / 3}-2[\cos \theta]_{\pi / 3}^{2 \pi / 3}-[\cos \theta]_{2 \pi / 3}^{\pi}\right. \\
&\left.\quad+[\cos \theta]_{\pi}^{4 \pi / 3}+2[\cos \theta]_{4 \pi / 3}^{5 \pi / 3}+[\cos \theta]_{5 \pi / 3}^{2 \pi}\right\} \\
&=\frac{2}{\pi} V_{d c} .
\end{aligned}
$$

The relationship between the dc line voltage and the $d q$-axis input phase voltages is derived by substituting (5) into (4)

$$
\begin{aligned}
{\left[\begin{array}{l}
\bar{V}_{d} \\
\bar{V}_{q}
\end{array}\right] } & =\sqrt{\frac{3}{2}}\left[\begin{array}{l}
\cos \phi \\
\sin \phi
\end{array}\right] \frac{2}{\pi} V_{d c} \\
& =\frac{\sqrt{6}}{\pi}\left[\begin{array}{l}
\cos \phi \\
\sin \phi
\end{array}\right] V_{d c} .
\end{aligned}
$$

\section{PRedictive HaRMONIC IDENTIFIER}

To understand the concept of the predictive identifier it is important to realize that the conventional identifier is inherently predictive. It assumes that the fundamental frequency component of the load current (voltage) measured some time in the past (a time set by the delay of the filter) is the same as the present value. This assumption is valid under steady-state conditions, but no longer valid under transient conditions where the fundamental frequency component is time-varying. It is clear that some sort of explicit prediction is necessary to operate an APF effectively under transient conditions.

In [9] Round and Mohan propose a time-domain artificial neural network (ANN) identifier for a shunt APF that works in the stationary reference frame. The identifier exhibits reasonable transient performance in that it tracks the changing fundamental with little delay. There is, however, a period of incomplete harmonic cancellation during the transient period. The computational demands of implementing such a identifier is high because it uses three ANNs each with a large number of inputs and neurons. 

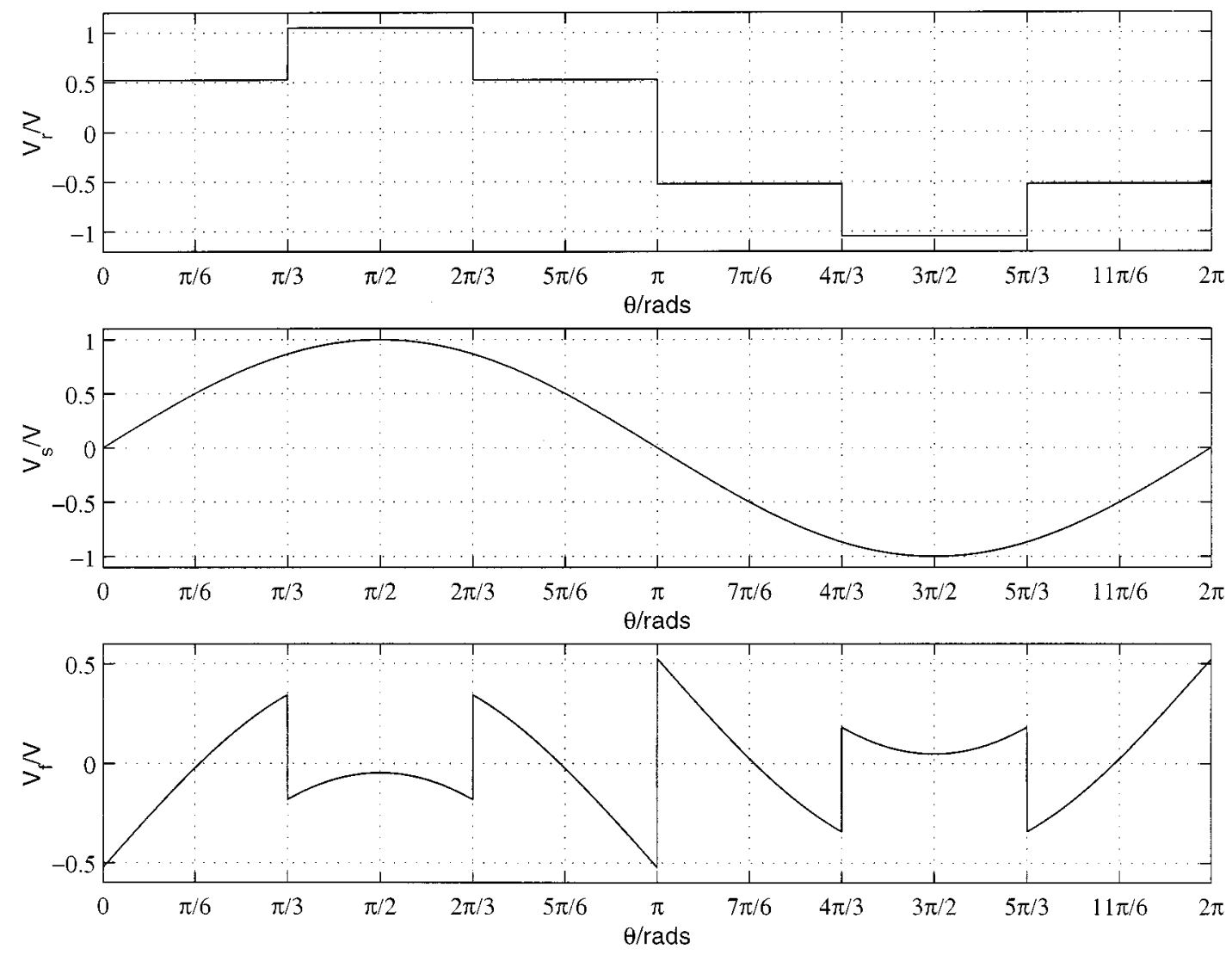

Fig. 6. Normalized ideal phase voltage waveforms for capacitive rectifier with series APF.

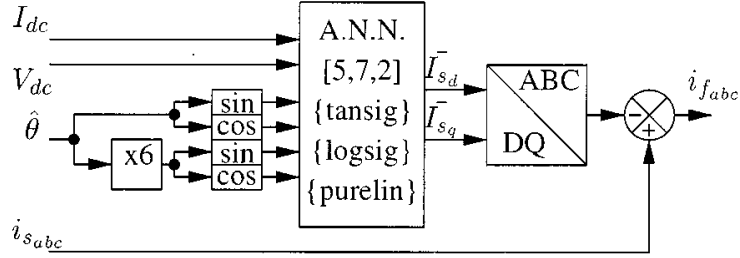

Fig. 7. Block diagram of ANN predictive harmonic identifier.

There is a growing trend in the use of ANN to control APFs. In many cases, an ANN is used to perform a task that is more simply performed with established digital signal processing techniques (DSP) such as fast Fourier transforms [10] or determining inverter switching patterns [11] where space vector modulation (SVM) is highly efficient.

A frequency-domain predictive APF identifier for a three-phase thyristor rectifier driven dc motor was proposed in [12]. The identifier exhibited excellent transient response but was sometimes problematic under steady-state conditions. Early simulation results for a predictive identifier similar to the one presented here have been presented for the single case of rectifiers with inductive de link filters [13].

The predictive identifier (Fig. 7) proposed here is for a threephase rectifier with inductive (capacitive) dc link filter. A feedforward ANN is used to estimate the mean $d q$-axis rectifier input currents (voltages) using the instantaneous dc link current and voltage. Information relating to the fundamental and sixth harmonic reference angles are also provided so that the fluctuation of dc current (voltage), assumed constant in Section III, can be

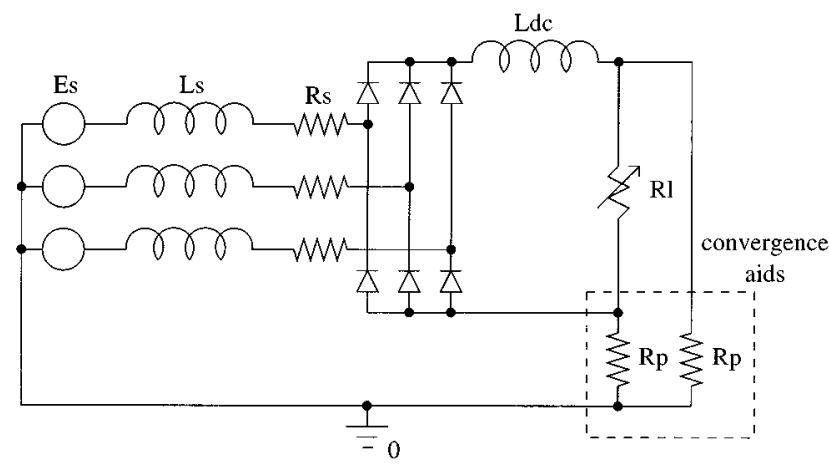

Fig. 8. PSpice simulation of rectifier with inductive dc filter and voltage source input.

TABLE I

PARAMETERS USED FOR SHUNT APF SIMULATION

\begin{tabular}{l|c}
\hline Peak Supply Voltage $\hat{V}_{s}$ & $270 \mathrm{~V}$ to $330 \mathrm{~V} @ 50 \mathrm{~Hz}$ \\
\hline Supply Inductance $L_{s}$ & $50 \mu \mathrm{H}$ \\
\hline Supply Resistance $R_{s}$ & $10 \mathrm{~m} \Omega$ \\
\hline DC Filter Inductance $L_{d c}$ & $100 \mathrm{mH}$ \\
\hline Load Resistance $R_{l}$ & $20 \Omega$ to $80 \Omega$ \\
\hline Parasitic Resistance $R_{p}$ & $1 G \Omega$ \\
\hline
\end{tabular}

accounted for. These estimated mean values are then subtracted from the instantaneous values to yield a cancellation reference. 

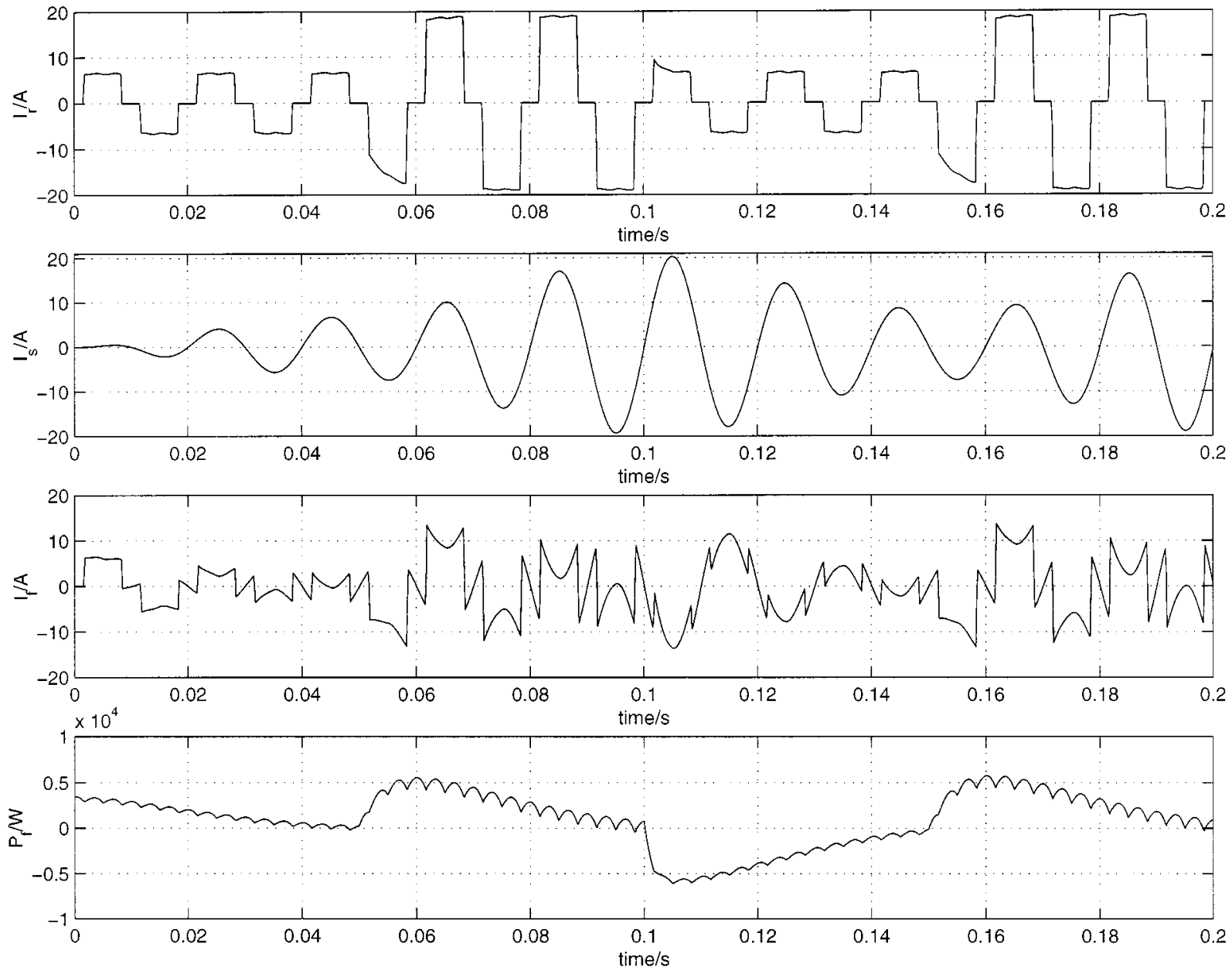

Fig. 9. Simulated load, estimated fundamental, and cancellation reference currents for conventional shunt APF.

The dc link current (voltage) is used as a basis for the prediction as it exhibits much less distortion than the $d q$-axis rectifier input currents. The sine and cosine functions of the sixth harmonic reference angles are included to give a continuous signal representing the periodicity of the dc link distortion. Similar information for the fundamental harmonic reference angle is included to allow the ANN to deal with unbalanced supply conditions.

The ANN used has the following configuration.

1) A hidden layer with five neurons with hyperbolic tangent sigmoid transfer function.

2) A second hidden layer with seven neurons with log sigmoid transfer function.

3) An output layer with two output neurons with linear transfer function.

A two hidden layer ANN is used as it is proven to be able to adapt to any arbitrary function [14], [15]. No delays or feedback were used within the ANN.

\section{A. Predictor Training}

The network is trained by presenting it with a large set of steady-state experimental input and target output data. The training is accomplished using the Levenberg-Marquardt (LM) back-propagation method [14], [15], in conjunction with the gradient descent with momentum (GDM) learning method
[14], [15] to update the ANN weight and bias values. The ANN performance is judged according to the mean squared error (MSE) between the actual ANN output and the target outputs. All training is carried out off-line in the MATLAB environment.

\section{B. Calculation of Target Data}

To generate the target output data for training the contemporary mean of the $d q$-axis rectifier distorted input current (voltage) is calculated using a noncausal moving average filter. Equation (7) is the linear difference equation representing the filter. This technique is chosen as it provides the best possible transient response. The filter uses both past and future data. This method can, therefore, only be used on data processed off-line

$$
\bar{X}(n)=\frac{1}{m} \sum_{k=-m / 2}^{m / 2-1} x(n-k)
$$

where

$\mathbf{n} \quad$ index of the present sample;

$\mathbf{m} \quad$ period of the input, in number of samples;

$\overline{\mathbf{X}}(\mathbf{n}) \quad$ mean value of the input at a specific sample index $\mathbf{n}$; 

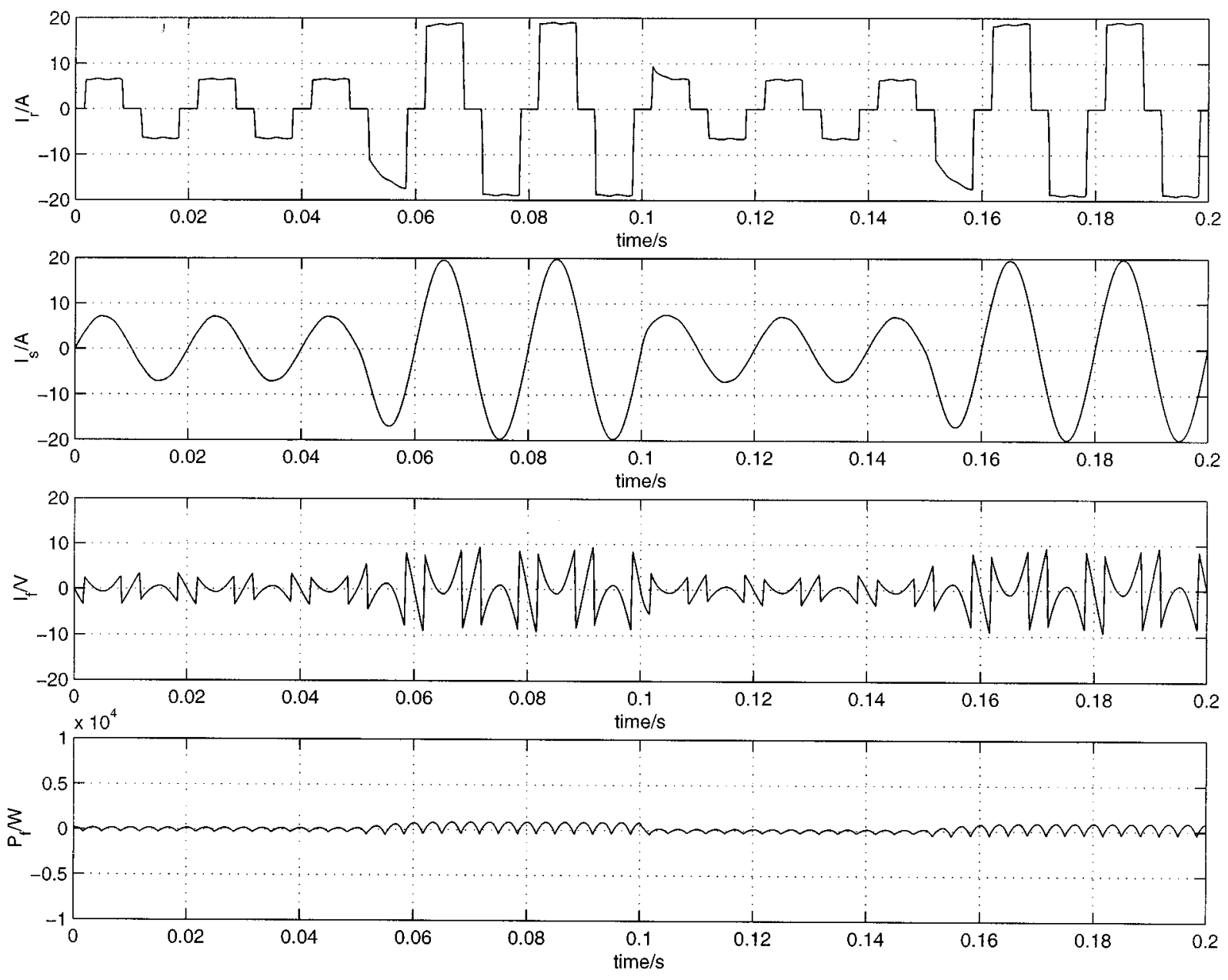

Fig. 10. Simulated load, estimated fundamental, and cancellation reference currents for predictive shunt APF.

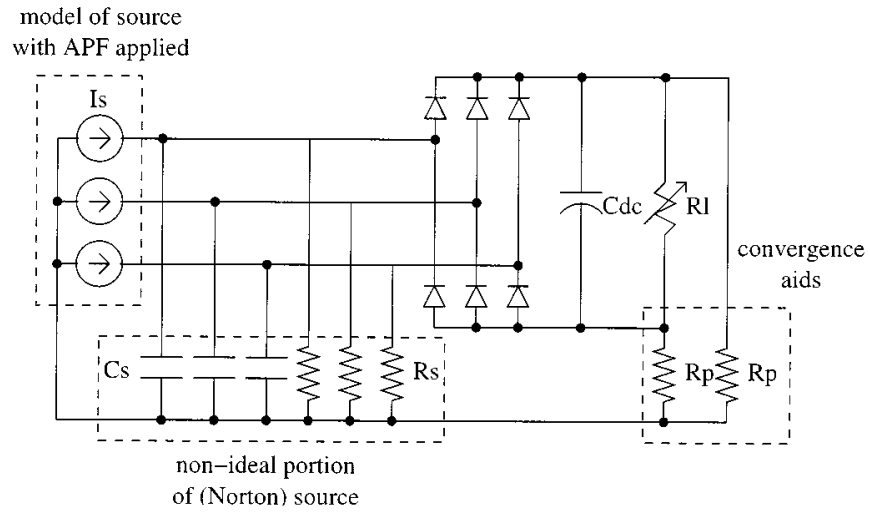

Fig. 11. PSpice simulation of rectifier with capacitive dc filter and current source input.

$$
\begin{array}{ll}
\mathbf{x}(\mathbf{n}-\mathbf{k}) & \text { input at sample index } \mathbf{n}-\mathbf{k} ; \\
\mathbf{k} & \text { sample index offset. }
\end{array}
$$

\section{Simulation}

In order to test the operation of the predictor models, several three-phase rectifiers were simulated in PSpice. Training and validation data was acquired for processing in MATLAB.
TABLE II

PARAMETERS USED FOR SERIES APF SIMULATION

\begin{tabular}{l|c}
\hline Peak Supply Current $\hat{I}_{s}$ & $9 A$ to $11 A @ 50 \mathrm{~Hz}$ \\
\hline Supply Capacitance $C_{s}$ & $5 n F$ \\
\hline Supply Resistance $R_{s}$ & $1 k \Omega$ \\
\hline DC Filter Capacitance $C_{d c}$ & $50 \mu F$ \\
\hline Load Resistance $R_{l}$ & $20 \Omega$ to $80 \Omega$ \\
\hline Parasitic Resistance $R_{p}$ & $1 G \Omega$ \\
\hline
\end{tabular}

\section{A. Shunt APF Simulation Results}

Fig. 8 shows the PSpice model of a three-phase rectifier with an inductive dc link filter. It was used to generate training and validation data for the shunt APF identifier of the configuration shown in Fig. 1. The load is a variable resistance. Line impedance is included in the model to ensure simulation convergence. The impedance is very small and hence the rectifier terminal voltages are very close to sinusoidal. In this mode of operation the rectifier behaves as if an shunt APF were in place. The parasitic resistance $R p$ is included to reference the dc link to the neutral line. It is necessary to reference the dc link to the 

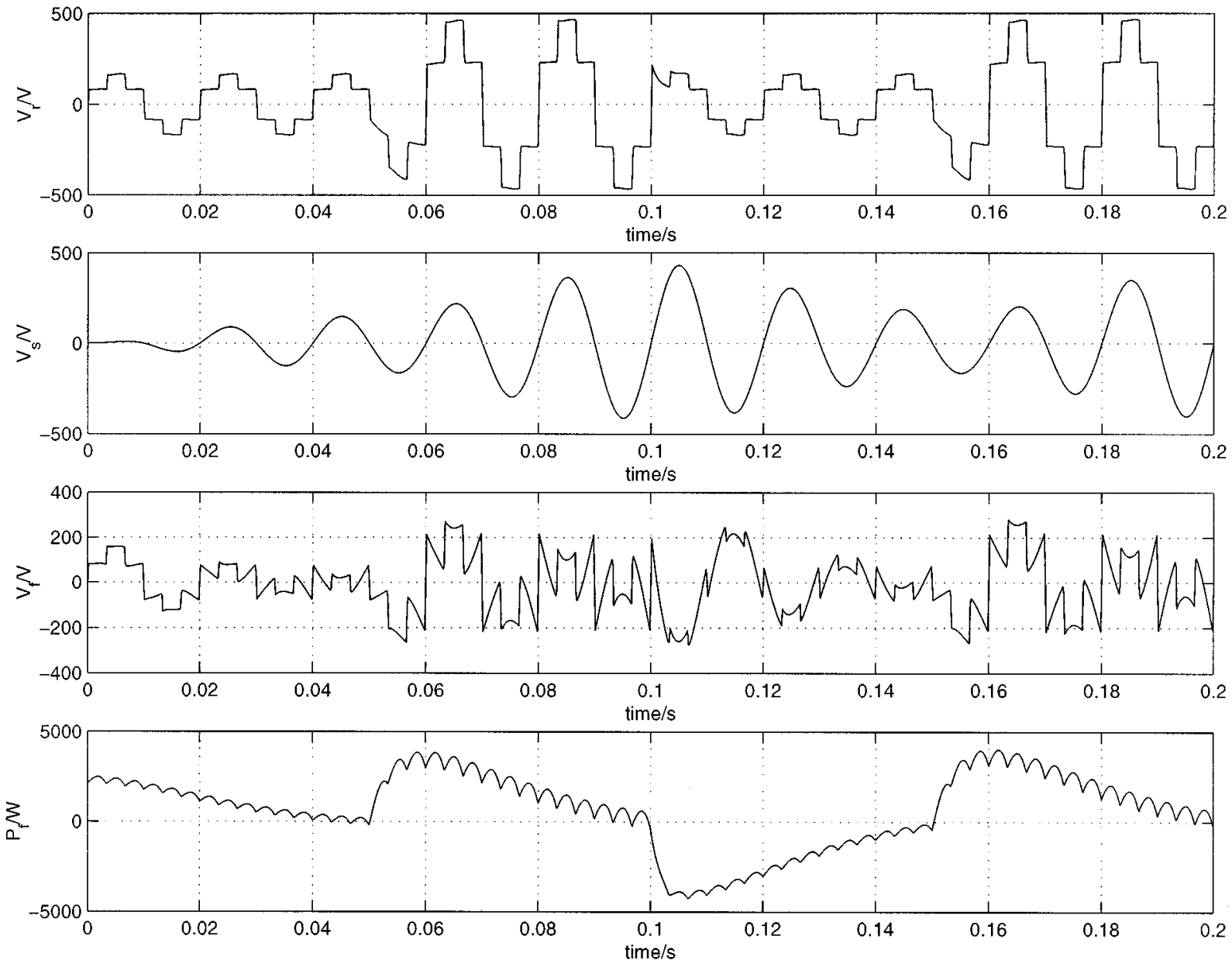

Fig. 12. Simulated load, estimated fundamental, and cancellation reference phase voltages for conventional series APF.

ac side to ensure convergence under starting conditions. Table I shows the parameters used.

Fig. 9 shows the performance of the conventional harmonic identifier for a shunt APF under transient conditions. There is a clear tracking delay in the estimated fundamental frequency component of the load current. This leads to a residual fundamental component in the cancellation reference current, which in turn causes a large real power flow in the APF.

In contrast, Fig. 10 shows the performance of the predictive shunt APF identifier over the same transient. The predictive identifier tracks the changing fundamental frequency component of the load current without delay and with negligible residual harmonic distortion. The cancellation reference is therefore accurate and gives no average power flow in the APF.

\section{B. Series APF Simulation Results}

Fig. 11 shows the PSpice model of a three-phase rectifier with a capacitive dc link filter under conditions of sinusoidal supply current. It was used to generate training and validation data for the series APF identifier of the configuration shown in Fig. 2. The currents were forced to be sinusoidal to train the predictor for a case where the APF was already active rather than training with a voltage source and non-APF case. The Norton equivalent of the line impedance shown in 8 is included to ensure convergence of the simulation. The impedance is very large and hence the rectifier input currents are very near sinusoidal. As with the previous simulation parasitic resistance is referenced the dc link to the ac side to ensure convergence under starting conditions. Table II shows the parameters used.

Fig. 12 shows the performance of the conventional harmonic identifier for a series APF under transient conditions. There is a clear tracking delay in the estimated fundamental frequency component of the load phase voltage. This leads to a residual fundamental component in the cancellation reference voltage, which in turn causes a large real power flow in the APF.

Fig. 13 shows the performance of the predictive series APF identifier over the same transient. The predictive identifier tracks the changing fundamental frequency component of the load phase voltage without delay and with negligible residual harmonic distortion. The cancellation reference is therefore accurate and gives no average power flow in the APF.

\section{EXPERIMENTAL RESULTS}

An experimental system was constructed in order to demonstrate the operation of the predictor in a realistic environment. The objectives were to show that

i) an ANN of the required size can be implemented in realtime; 

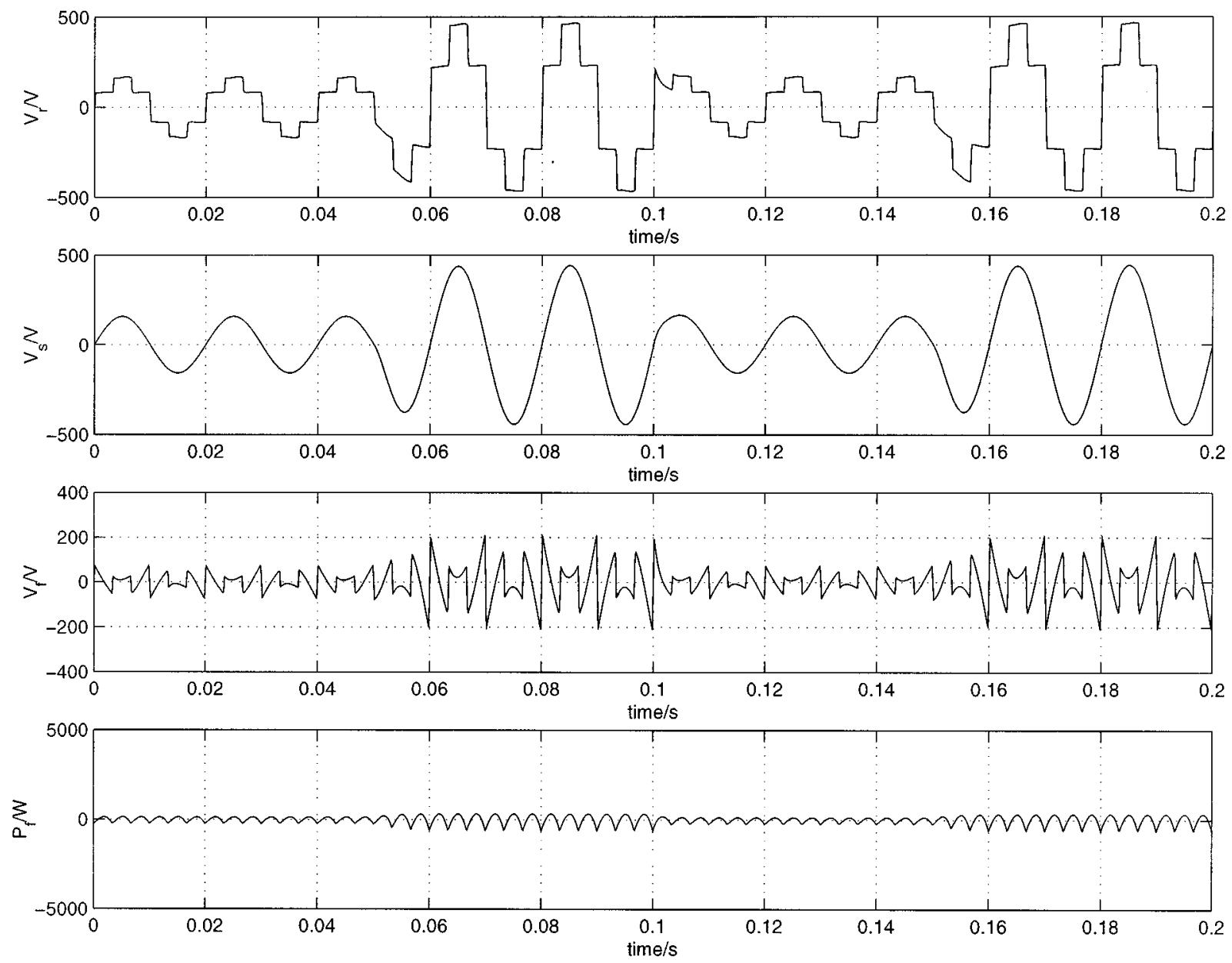

Fig. 13. Simulated load, estimated fundamental, and cancellation reference phase voltages for conventional shunt APF.

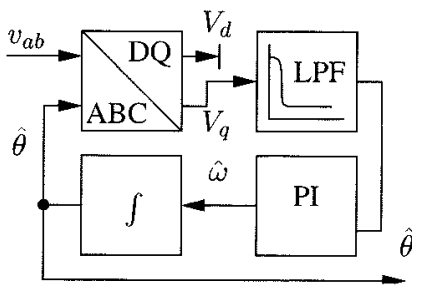

Fig. 14. Block diagram of digital three-phase phase-locked loop.

ii) the quantization of the ANN and the look-up tables of the activation functions are acceptable approximations;

iii) that the predictor can work in nonideal circumstances with overlap in commutation, line voltage distortion and rectifier dc-side current ripple.

The results concentrate on the harmonic identifier and do not include the functioning of the APF.

The test system consisted of a three-phase diode bridge rectifier with a $230 \mathrm{mH}$ dc-side inductance and variable resistance. The rectifier was connected to a $415 \mathrm{~V} 50 \mathrm{~Hz}$ supply via a VARIAC. Real-time processing is provided by a $50 \mathrm{MHz}$ TMS320C44 digital signal processor. Analogue input is achieved by means of 12-b ADCs. The DSP board is housed in a Pentium 200MMX IBM compatible PC host with 128 MB of RAM. The setup offers fast bidirectional data exchange between PC and DSP platforms via dual-port RAM.
A suite of software distributed over DSP and PC platforms provides real-time processing and data-acquisition. The implementation of the predictive identifier was optimized for speed by use of interpolated lookup tables for the activation functions of the ANN. The resulting execution time allowed for a sampling frequency of $6.4 \mathrm{kHz}$, corresponding to 128 samples per $50 \mathrm{~Hz}$ mains cycle.

\section{A. Three-Phase PLL}

Implementation of the predictive identifier requires that an accurate estimate of the mains reference angle is known. To this end a discrete-time three-phase phase-locked loop (PLL) [16]-[18] is implemented on the DSP. Fig. 14 shows a block diagram of the PLL. The regulator works by adjusting the estimated frequency until the mean value of the $q$-axis voltage (obtained by the LPF) is zero. The estimated frequency is then integrated to yield the estimated mains reference angle. This is the angle used to perform the $d q$ transformation on the input three-phase voltage.

The PLL was tested on a variety of harmonically distorted and unbalanced three-phase networks and successfully locked on to the fundamental phase and frequency with a high degree of accuracy. Fig. 15 shows an oscilloscope plot of the PLL successfully locked onto an extremely distorted and unbalanced three-phase network. 


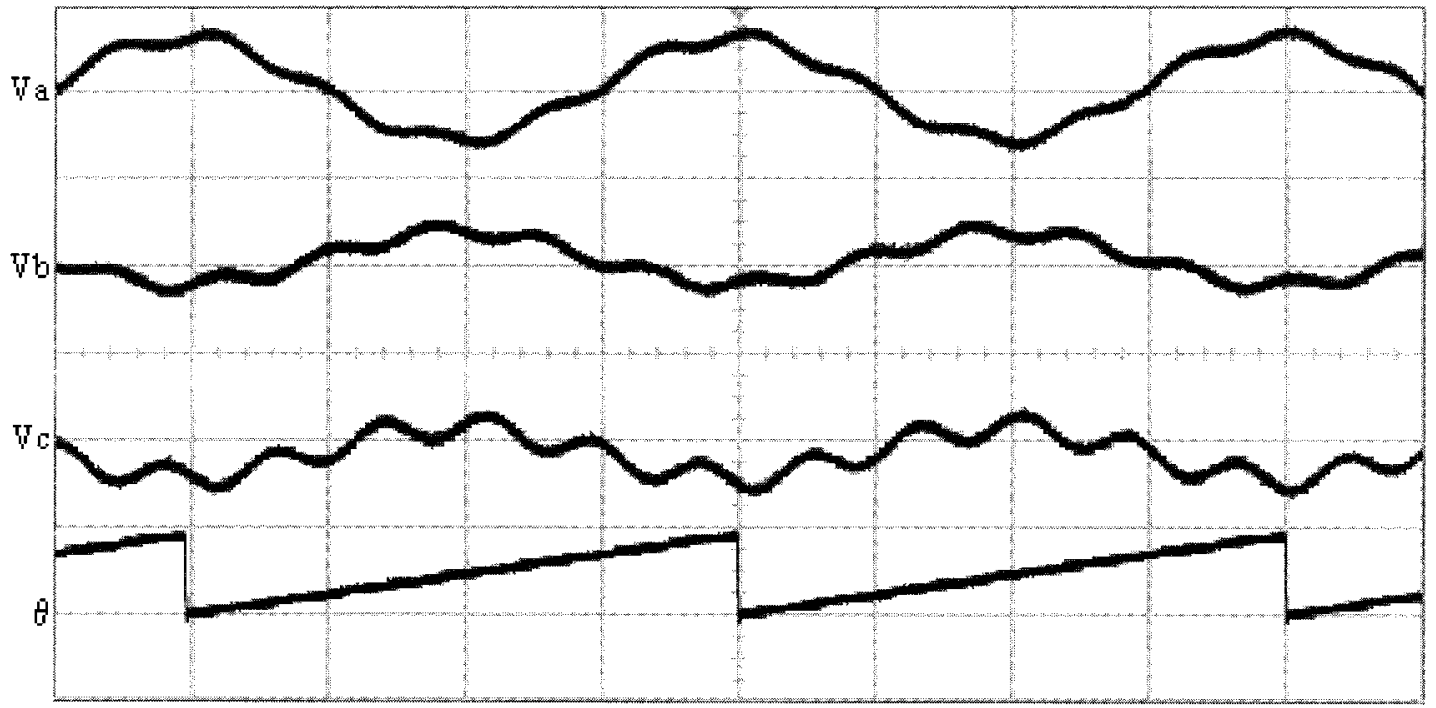

Fig. 15. Distorted phase voltage input to PLL and estimated reference angle. ABC phase voltages, $V_{a}, V_{b}, V_{c}(150 \mathrm{~V} / \mathrm{div})$ estimated angle, $\theta(2 \pi \mathrm{rad} / \mathrm{div})$. Timebase $5 \mathrm{~ms} / \mathrm{div}$.

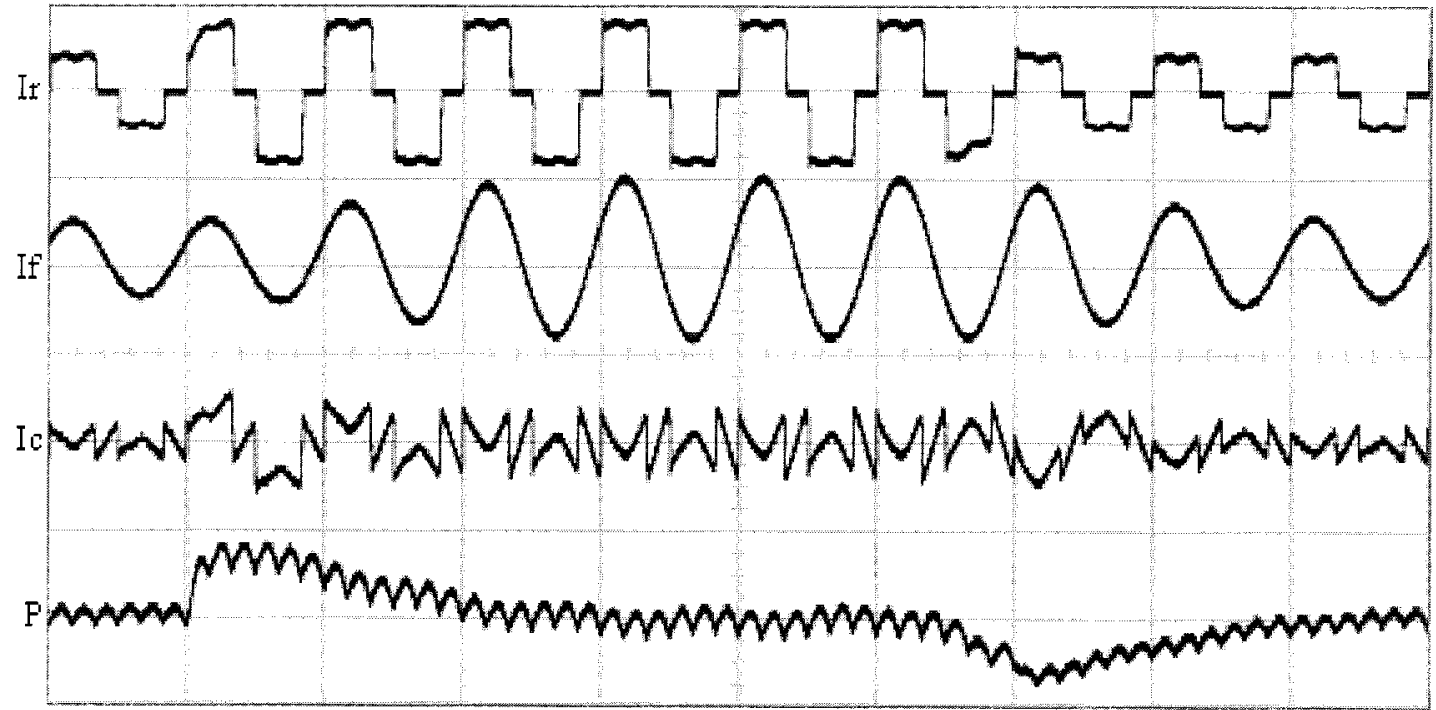

Fig. 16. Conventional harmonic identifier experimental results. Rectifier current, $I_{r}$, estimated fundamental current, $I_{f}$ and cancellation current, $I_{c}(5 \mathrm{~A} /$ div). APF power, $P(750 \mathrm{~W} / \mathrm{div})$. Timebase $20 \mathrm{~ms} / \mathrm{div}$.

\section{B. Shunt APF Experimental Results}

Fig. 16 shows the performance of the conventional shunt identifier under transient conditions. The conventional identifier exhibits a substantial delay in tracking the changing fundamental frequency component of the rectifier input current. This results in a residual fundamental component in the cancellation reference and consequently real power flow in the APF.

Fig. 17 shows the performance of the predictive shunt identifier under the same transient conditions. The predictive identifier tracks the changing fundamental frequency component of the rectifier input current without delay. This results in an ac- curate cancellation reference. The average APF power is zero throughout the transients.

The experimental results are in close agreement with the simulation results shown in Fig. 9 for the conventional identifier and Fig. 10 for the predictive identifier.

\section{CONCLUSIONS}

In this paper, a novel predictive distortion identifier for an APF was proposed in order to overcome the tracking delays experienced with conventional APFs utilizing time or frequency domain filtering techniques. The identifier employed an artifi- 


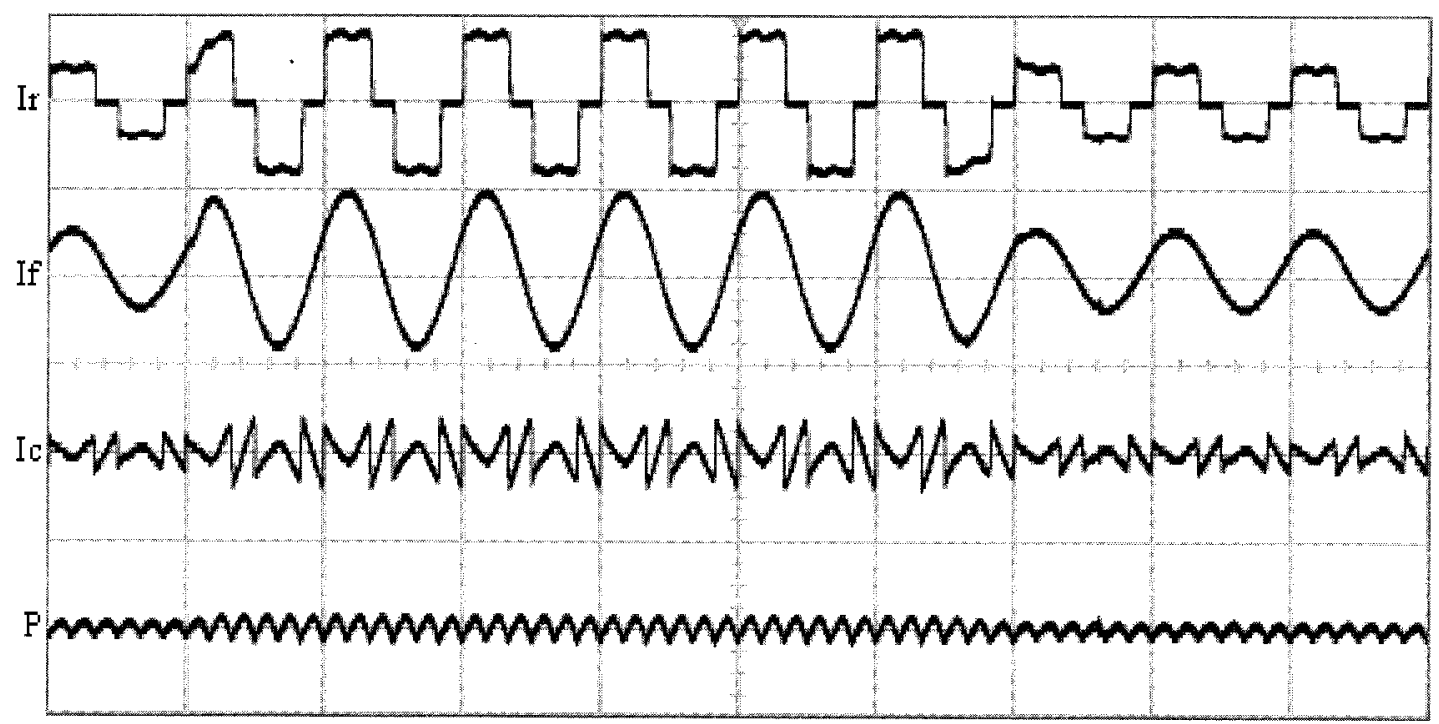

Fig. 17. Predictive harmonic identifier experimental results. Rectifier current, $I_{r}$, estimated fundamental current, $I_{f}$ and cancellation current, $I_{c}(5 \mathrm{~A} / \mathrm{div})$. APF power, $P(750 \mathrm{~W} / \mathrm{div})$. Timebase $20 \mathrm{~ms} / \mathrm{div}$.

cial neural network to predict the mean $d q$-axis input to an uncontrolled diode bridge rectifier. It was adapted for both series and shunt APF configurations.

A relationship between the dc link current (voltage) and mean $d q$-axis current (voltage) of an uncontrolled three-phase diode bridge rectifier was derived for ideal conditions in order to validate the basis for predicting the mean $d q$-axis current (voltage) from its instantaneous DC counterpart.

Simulation results with PSpice and MATLAB showed good steady-state performance for both series and shunt predictive harmonic identifiers. The conventional identifier exhibited poor transient response, due to the delays incurred in the filtering process. This in turn led to a fundamental frequency component appearing in the cancellation reference and would consequently result in real power flow in the APF. The predictive identifier exhibited no delay in tracking the fundamental and showed negligible residual harmonic distortion. This resulted in an accurate cancellation reference and zero average real power flow in the APF.

An experimental system was implemented in order to validate the results of the predictive identifier for a shunt APF. The identifier showed good steady-state and excellent transient response. The agreement between simulation and experimental results was very good. This demonstrates that

a) it is realistic to run an ANN in real time at the sample rates required

b) the quantization of the network activation function look-up tables is not a problem

c) the ANN trained on simulation data can generalize to a real case with nonideal features such as commutation overlap and mains voltage distortion.

The implications of improved transient response of an APF identifier are reduced power rating, energy storage component size and control system bandwidth. This results in reduced implementation cost of an APF.

\section{REFERENCES}

[1] F. Z. Peng, "Application issues of active power filters," IEEE Ind. Applicat. Mag., vol. 4, pp. 21-30, Sept./Oct. 1998.

[2] S. Bhattacharya, T. M. Frank, D. M. Divan, and B. Banerjee, "Active filter system implementation," IEEE Ind. Applicat. Mag., vol. 5, pp. 47-63, Sept./Oct. 1998.

[3] L. Pittorino, A. Horn, and J. Enslin, "Power theory evaluation for the control of an active power filter," in Proc. IEEE AFRICON'96, vol. 2, June 1996, pp. 676-681.

[4] S.-G. Jeong and M.-H. Woo, "D.s.p. based active power filter with predictive current control," in Proc. IEEE Ind. Electron., Contr. Instrum. IECON'95, vol. 1, Sept. 1995, pp. 645-650.

[5] L. Benchaita, S. Saadate, and A. Salem nia, "A comparison of voltage source and current source shunt active filter by simulation and experimentation," IEEE Trans. Power Syst., vol. 2, pp. 642-647, May 1999.

[6] C. V. Nunez-Noriega and G. G. Karady, "Five step-low frequency switching active power filter for network harmonic compensation in substations," IEEE Trans. Power Delivery, vol. 4, pp. 1298-1303, Oct. 1999.

[7] S. Kim and P. N. Enjeti, "Control strategies for active power filter in three-phase four-wire systems," in Proc. APEC'00 Conf., vol. 1, 2000, pp. $420-426$.

[8] E. Clarke, Circuit Analysis of A.C. Power Systems, 1st ed. New York: Wiley, 1943, vol. I and II

[9] S. D. Round and N. Mohan, "Comparison of frequency and time domain neural network controllers for an active power filter," in Proc. Ind. Electron., Contr. Instrum., vol. 2, 1993, pp. 1099-1104.

[10] N. Pecharanin, H. Mitsui, and M. Sone, "Harmonic detection by using neural network," in Proc. IEEE Int. Conf. Neural Networks, vol. 2, 1995, pp. 923-926.

[11] A. Elmitwally, S. Abdelkader, and M. El-Kateb, "Neural network controlled three-phase four-wire shunt active power filter," Proc. Inst. Elect. Eng., vol. 2, no. 147, pp. 87-92, 2000.

[12] Z. Lu and T. Green, "Neural network based predictive control strategy of active power filter for electric drives," in Proc. IEEE Power Electron. Variable Speed Drives PEVD'98 Conf., vol. 7, 1998, pp. 287-291.

[13] J. H. Marks and T. C. Green, "Predictive control of active power filters," in Proc. IEEE Power Electron. Variable Speed Drives PEVD'OO Conf., vol. 1, 2000, pp. 18-23.

[14] K. Gurney, An Introduction to Neural Networks. London, U.K.: UCL, 1997.

[15] R. Beale and T. Jackson, Neural Computing an Introduction. Philidelphia, PA: IOP, 1990.

[16] N. Bruyant and M. Machmou, "Simplified digital-analogical control for shunt active power filters under unbalanced conditions," Proc. Inst. Elect. Eng., vol. 456, no. 7, pp. 11-16, Sept. 1998.

[17] V. Kaura and V. Blasko, "Operation of a phase locked loop system under distorted utility conditions," in Proc. Appl. Power Electron. Conf. Expo. (APEC'96), vol. 2, July 1996, pp. 703-708. 
[18] S.-K. Chung, "Phase-locked loop for grid connected three-phase power conversion systems," Proc. Inst. Elect. Eng., vol. 147, pp. 213-219, May 2000 .

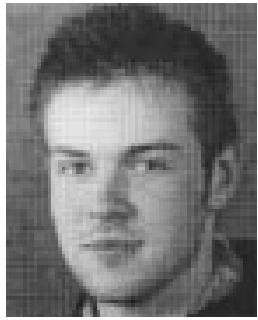

John H. Marks (S'02) received the B.Eng. degree (first class honors) in mechatronics from the University of Leeds, U.K., in 1997 and is currently pursuing the Ph.D. degree in electrical engineering in the Department of Electrical and Electronic Engineering, the Imperial College of Science, Technology, and Medicine, London, U.K.

His research interests include power quality, adaptive neural control systems, and power electronics.

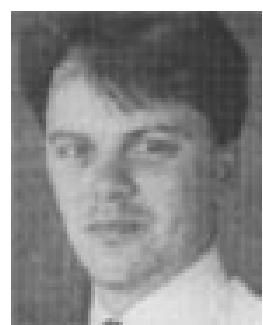

Tim C. Green (M'89) received the B.Sc. degree (with first class honors) from Imperial College of Science, Technology, and Medicine, London, U.K. in 1986 and the Ph.D. degree from Heriot-Watt University (HWU), Edinburgh, U.K. in 1990, both in electrical engineering.

He was at HWU until 1994 and is now a Senior Lecturer at Imperial College and member of the Control and Power Research Group. He has research interests in power quality and power delivery covering active power filters, unified power flow controllers, embedded generation, and un-interruptable supplies. He also pursues series/parallel use of power modules for frequency spectrum improvement and the design of integrated high speed drives systems. 